Thus the behaviour of ultra-violet rays and the behaviour of electromagnetic waves both yield, although in different ways, evidence of ionization in the upper atmosphere.

55, King Street, South, J. R. Ashworth. Rochdale. July 24.

${ }^{1}$ Appleton, Phil. Mag., Ser. 7, 27, 144 (1939).

${ }^{2}$ Pettit, E., Astro. J., 75, 185 (1932).

${ }^{3}$ Ashworth, Proc. Manchester Lit. and Phil. Soc., 83, 81 (1939); and Pettit, loc. cit.

\section{After-burning of Carbon Monoxide: Spectroscopic Evidence for Abnormal Dissociation}

IN recent papers ${ }^{1,2}$ I have discussed the cause of the after-burning and latent energy of the carbon monoxide flame in relation to the flame spectrum, and have given estimates of the lives of the vibrationally activated molecules which are formed. It was also suggested that the high vibrational energy of the newly formed molecules might result in a large amount of dissociation. Since I communicated these papers, David, Leah and Pugh ${ }^{3}$ have published some experiments on latent energy and dissociation in flame gases which give striking support to my suggestion.

The theory of the later stagas of the combustion of carbon monoxide may be summarized as follows :

(a) Since the dissociation products of a normal carbon dioxide molecule are not normal $\mathrm{CO}\left({ }^{1} \Sigma\right)+$ $\mathrm{O}\left({ }^{3} P\right)$, but electronically excited $\mathrm{CO}\left({ }^{3} \Omega\right)+\mathrm{O}\left({ }^{3} P\right)$, the formation of normal carbon dioxide from normal carbon monoxide and oxygen must require an electronic rearrangement in the newly formed carbon dioxide molecule.

(b) The excited state of carbon dioxide, before the electronic rearrangement, is probably triangular, while the normal carbon dioxide molecule is known to be linear. Hence the electronic rearrangement, whether occurring by radiation or on collision, will result in the newly formed molecule acquiring a large amount of vibrational energy owing to the sudden change in shape.

(c) This excess vibrational energy will, if the gases are pure, persist for an appreciable fraction of a second. In the presence of moisture, however, the excess energy will be quickly transformed into thermal energy.

(d) For the carbon dioxide molecule the transverse vibration $v_{2}$ and the symmetrical vibration $v_{1}$ have frequencies which satisfy the relation $v_{1}=2 v_{2}$ to a very close approximation. This degeneracy may result in resonance between the two vibrations and transfer of energy from one form to another, and thus may result in dissociation of the molecule if its vibrational energy content is sufficiently high.

This theory appears to explain many of the peculiarities of the combustion of carbon monoxide, such as the effect of moisture on flame speed and flame temperature, the effect of drying in increasing the infra-red radiation from explosions, and the failure to observe the infra-red band at $14.9 \mu$.

In the recent experiments by David and his colleagues it has been shown that temperature measurements of flames made with wires coated with quartz indicate an abnormally high dissociation of the $\mathrm{CO}_{2}$ molecules, this being greatly in excess of the dissociation expected at the flame temperature. If the newly formed carbon dioxide molecules are vibrationally activated, as indicated by the theory above, they may be regarded as having a very high effective vibrational temperature, this being much greater than their effective translational temperature. Since it is the vibrational temperature which determines the dissociation of the molecules, it is to be expected that the vibrationally excited molecules formed by the combustion will show an abnormally high dissociation.

The afterglow of carbon dioxide in a discharge tube ${ }^{5}$ and the after-burning should both be regarded as manifestations of this dissociation, the usual flame spectrum being emitted during the recombination processes following the dissociation. The emission of light during the after-burning or afterglow should not be regarded as due to emission from activated (metastable) molecules, which have not sufficient energy to give a spectrum in the ultra-violet ${ }^{6}$, but as due to the flame of gases which are recombining following the abnormal dissociation. Increase of pressure will increase the light emission from the after-burning gases by altering the dissociation equilibrium; Prof. A. C. G. Egerton points out that this is supported by observations of the inereased luminosity at the centre during explosions in closed vessels when the pressure wave travels back through the burnt gases.

Chemical Technology Dept.,

A. G. GaYDon.

$$
\begin{gathered}
\text { Imperial College, } \\
\text { London, s.W.7. } \\
\text { July } 24 .
\end{gathered}
$$

${ }^{1}$ Gaydon, A. G., Proc. Roy. Soc., A, 176, 505 (1940).

${ }^{2}$ Gaydon, A. G., Proc. Roy. Soc., A, 173, 61 (1941).

${ }^{3}$ David, W. T., Leah, A. S., and Pugh, B., Phil. Mag., 31, 156 (1941).

${ }^{4}$ Garner, W. E., Johnson, C. H., and Saunders, S. W., Nature, 117, 790 (1926).

s Fowler, A., and Gaydon, A. G., Proc. Roy. Soc., A, 142, 362 (1933).

${ }^{\circ}$ Egerton, A., and Ubbelohde, A. R., NAture, 134, 848 (1934).

\section{"White Horses"}

THE behaviour of secondary waves riding on' the larger primary ones does not seem to have been taken into account in the theory of wind-formed sea waves. From the geometry of trochoids, surface water particles at the troughs on either side of the crest of a secondary wave will close up when this wave is riding on the crest of a primary one, and open out at its trough. The energy of the secondary wave will squeeze up and disperse accordingly ; and height, and so steepness, will be affected not only on that account, but also because the relationship of height to energy will change owing to the vertical acceleration of the water caused by the primary wave.

Thus, if $L, H, S, E$ denote length, height, steepness and energy per sq. ft. of water surface of the primary wave, $S$ being taken as $H \div L$, and $l, h, s, e$ apply to the secondary wave, $l$ will range from $l \times$ $(1-\pi S)$ at crest, to $l \times(1 \times \pi S)$ at trough ; $e$ from $e \div(1-\pi S)$ to $e \div(1+\pi S)$; and $W$ from $64 \times(1-\pi S)$ to $64 \times(1+\pi S) \mathrm{lb}$. per cu. $\mathrm{ft}$. Therefore, as $e=W \cdot h^{2} \div 8, h$ will range from $h \div$ $(1-\pi S)$ to $h \div(1+\pi S)$, and $s$ from $s \div$ $(1-\pi S)^{2}$ to $s \div(1+\pi S)^{2}$. It will be noticed that this deformation is independent of the steepness of the secondary wave, and of whether that wave is moving with or against the primary one. 